\title{
Diacronie
}

Studi di Storia Contemporanea

$N^{\circ} 35,3 \mid 2018$

Gli strumenti di Clio

\section{Commento di Carmelo Albanese a Un'età contro la storia. Saggio sulla rivoluzione del XXI secolo}

\section{Carmelo Albanese}

\section{(2) OpenEdition}

Journals

\section{Edizione digitale}

URL: http://journals.openedition.org/diacronie/9034

DOI: 10.4000/diacronie. 9034

ISSN: 2038-0925

\section{Editore}

Association culturelle Diacronie

\section{Notizia bibliografica digitale}

Carmelo Albanese, "Commento di Carmelo Albanese a Un'età contro la storia. Saggio sulla rivoluzione del XXI secolo », Diacronie [Online], № 35, 3 | 2018, documento 7, Messo online il 29 septembre 2018, consultato il 22 avril 2019. URL : http://journals.openedition.org/diacronie/9034; DOI : 10.4000/ diacronie.9034 


\section{Diacronie}

Studi di Storia Contemporanea

\section{$35,3 / 2018$}

Gli strumenti di Clio: uomini, luoghi e teorie della storia dalla tradizione critica alla comunicazione digitale

\section{Commento di Carmelo Albanese a Un'età contro la storia. Saggio sulla rivoluzione del XXI secolo}

\section{Carmelo ALBANESE}

Per citare questo articolo:

ALBANESE, Carmelo, «Commento di Carmelo Albanese a Un'età contro la storia. Saggio sulla rivoluzione del XXI secolo», Diacronie. Studi di Storia Contemporanea : Gli strumenti di Clio: uomini, luoghi e teorie della storia dalla tradizione critica alla comunicazione digitale, 35, 3/2018, 29/09/2018,

URL: <http://www.studistorici.com/2018/09/29/albanese_numero_35/ >

Diacronie Studi di Storia Contemporanea $\rightarrow$ http://www.diacronie.it

Rivista storica online. Uscita trimestrale.

redazione.diacronie@hotmail.it

Comitato di direzione: Naor Ben-Yehoyada - João Fábio Bertonha - Christopher Denis-Delacour - Maximiliano Fuentes Codera Anders Granås Kjøstvedt - John Paul Newman - Deborah Paci - Niccolò Pianciola - Spyridon Ploumidis - Wilko Graf Von Hardenberg

Comitato di redazione: Jacopo Bassi - Luca Bufarale - Gianluca Canè - Fausto Pietrancosta - Alessandro Salvador - Matteo Tomasoni Diritti: gli articoli di Diacronie. Studi di Storia Contemporanea sono pubblicati sotto licenza Creative Commons 3.0. Possono essere riprodotti e modificati a patto di indicare eventuali modifiche dei contenuti, di riconoscere la paternità dell'opera e di condividerla allo stesso modo. La citazione di estratti è comunque sempre autorizzata, nei limiti previsti dalla legge. 


\title{
7/ Commento di Carmelo Albanese a Un'età contro la storia. Saggio sulla rivoluzione del XXI secolo
}

\author{
Carmelo ALBANESE
}

Carmelo Albanese nel suo commento al saggio di Giuseppe Carlo Marino su globalizzazione e crisi del "pensare storico" ripercorre i tratti fondamentali dell'analisi proposta dallo studioso inerente il rapporto tra dinamiche economico-sociali, mercificazione del tempo, affermazione delle nuove tecnologie dell 'informazione, offrendoci una lettura personale delle conseguenze politico-culturali e dei connessi effetti sulle modalità di ri-definizione del dibattito storiografico nel XXI secolo.

\section{Introduzione}

Giuseppe Carlo Marino è uno storico di lungo corso. Già docente all'Università di Palermo, i suoi studi hanno riguardato la storia sociale e politica italiana tra Otto e Novecento: una vasta produzione bibliografica prevalentemente orientata ad analizzare contesti, soggetti e protagonisti del conflitto sociale, eppure attraversata da un "filo rosso" costituito da una sorta di insopprimibile interesse ad identificare e definire le «forme ideologiche» e le pratiche di potere della borghesia italiana ${ }^{1}$. Da questi indirizzi di ricerca esula, in apparenza, il volume che dà alle stampe all'inizio del nuovo secolo con l'ambizioso titolo Eclissi del principe e crisi della storia. Apogeo e tramonto della democrazia rivoluzionaria nel XXI secolo: una ricostruzione "biografica" del "principe" - come egli stesso afferma ${ }^{2}$-, ovvero una indagine sulla evoluzione del "potere

\footnotetext{
${ }^{1}$ Cfr. MARINO, Giuseppe Carlo, La formazione dello spirito borghese in Italia, Firenze, La Nuova Italia, 1974, da cui è tratta la citazione, p. IX; ID., Autoritratto del Pci staliniano, Roma, Editori Riuniti, 1992; ID., Guerra fredda e conflitto sociale in Italia, Caltanissetta-Roma, Sciascia Editore, 1994; ID., La repubblica della forza, Milano, Franco Angeli, 1995. Analoga propensione si manifesta in modo ancor più evidente nelle indagini settoriali sulla storia della Sicilia. Cfr. ad esempio ID., Il meridionalismo della destra storica e l'inchiesta parlamentare del 1867 su Palermo, Palermo-São Paulo, Ila Palma, 1971; ID., Partiti e lotta di classe in Sicilia: da Orlando a Mussolini, Bari, De Donato, 1976; ID., Storia del separatismo siciliano (1943-1947), Roma, Editori Riuniti, 1979; ID., Storia della mafia, Roma, Newton\&Compton, 1998.

${ }^{2}$ ID., Eclissi del principe e crisi della storia. Apogeo e tramonto della democrazia rivoluzionaria nel XXI secolo, Milano, Franco Angeli, 2000, p. 7.
} 
democratico", dalla rivoluzione francese a quella russa, che gli eventi epocali del quadriennio 1989-1993 hanno fatto tramontare, con un focus specifico sul Partito comunista italiano nel suo «faticoso tragitto ideale dai vincoli dello stalinismo alla piena valorizzazione del pensiero di Antonio Gramsci» per dar vita a una inedita forma di "principe" «nazional-popolare». Nel tracciare le linee di fondo di un periodo estremamente lungo, l'autore mette in luce le problematiche che il combinato tra il crollo del "socialismo reale" e il dispiegarsi nella più avanzata contemporaneità della "rivoluzione elettronico-informatica" ha comportato per l'intera tradizione democratica occidentale, sia di matrice socialista che liberale (dall'abbandono dei classici riferimenti ideali alla disgregazione sociale), in uno scenario in cui l'estensione del dominio del mercato persino alla sovranità di Stati e parlamenti cammina di pari passo con l'incremento esponenziale di incertezza e barbarie. Il saggio che viene sottoposto alla nostra attenzione - in una forma editoriale, quella dell'e-book, forse un po' troppo sacrificata per la rilevanza e l'interesse dei temi esposti - porta a maturazione queste suggestioni.

Utilizzando con meticolosità le categorie di lettura della scienza della storia sviluppate da Karl Marx - di cui rivendica per intero la validità interpretativa e la necessaria utilizzazione per la comprensione del presente ${ }^{4}$-, Marino sviluppa le principali direttrici del volume del 2000, e verificando, a distanza di quasi vent'anni, le ricadute profonde - sul piano antropologico, economico, politico e sociale - che hanno avuto processi allora solo in incubazione, presagisce l'inizio di un nuovo "tempo storico" senza "memoria", annientata dalla a-temporalità della cultura tecnologica che informa ormai la vita. Tra le vittime di una svolta così significativa vi sarebbe il lavoro storiografico e il mestiere di storico, sempre più derubricato al ruolo superfluo di "conoscitore di dettagli e fatti curiosi" ma reso incapace di produrre «sintesi interpretative idonee a mettere in luce [...] l'avanzata della "ragione", nonché della libertà e dell'humanitas, sull'itinerario della civiltà» ${ }^{5}$.

Il principio dialettico è al centro della trattazione. Certo, l'individuazione di una categoria attorno alla quale far gravitare l'intera narrazione è funzionale alla necessaria sintesi che il

\footnotetext{
${ }^{3}$ Ibidem, pp. 178-240, le citazioni sono alle pp. 178-179.

${ }^{4}$ Necessità già avvertita da HOBSBAWM, Eric J., Come cambiare il mondo. Perché riscoprire l'eredità del marxismo, Milano, Rizzoli, 2011, e ormai definitivamente affermatasi in ambito accademico e non solo, come evidenzia la mole di studi sul pensiero marxista pubblicati quest'anno in occasione del bicentenario della nascita del filosofo di Treviri. A titolo puramente esemplificativo, cfr. il volume collettaneo curato da CARIOTI, Antonio, Karl Marx vivo o morto? Il profeta del comunismo duecento anni dopo, Milano, Solferino, 2018.

${ }^{5}$ MARINO, Giuseppe Carlo, Un'età contro la storia. Saggio sulla rivoluzione del XXI secolo, Palermo, Università degli Studi di Palermo, Dipartimento di Scienze Politiche e delle relazioni internazionali (DEMS), 2017, p. 51. Su tali questioni è in corso da tempo un intenso dibattito internazionale, originato dalla pubblicazione in open access, nel 2014, del pamphlet di GUIDI, Jo, ARMITAGE, David, The History Manifesto, Cambridge, Cambridge University Press, URL: <https://www.cambridge.org/core/books/the-historymanifesto/AC1A1EC711AE91A4F9004E7582D79AFD > [consultato il 13 luglio 2018], tradotto in italiano nel 2016 da Donzelli (Manifesto per la storia. Il ruolo del passato nel mondo d'oggi). Due importanti confronti su questo saggio sono stati ospitati dalla rivista Memoria e ricerca, 1/2016, pp. 97-126, e dal sito della rivista Ricerche di storia politica, 13 ottobre 2015, URL: < http://www.ricerchedistoriapolitica.it/tavole-rotonde-econvegni/historians-of-the-world-unite-tavola-rotonda-su-the-history-manifesto-di-jo-guldi-e-davidarmitage-2/ > [consultato il 13 luglio 2018], ai quali rinvio.
} 
formato editoriale impone, e dunque alla esigenza di ricondurre ai suoi assi fondamentali - o ritenuti tali - una storia naturalmente lunga, complessa e oltremodo articolata. In ogni caso, nelle ventuno parti in cui è suddiviso il saggio, l'affermazione o il "tradimento" della logica propria del “tempo storico" scandiscono il percorso evolutivo dell'uomo nel mondo, significandone gli snodi cruciali.

Nei primi quattro pregnanti paragrafi diremmo "introduttivi", l'autore, adottando un inedito (trattandosi di uno storico) punto di osservazione filosofico - che per alcuni versi, come egli stesso riconosce, tende a «eccedere in "astrattezza" teoretica»" ${ }^{6}$, ripercorre le tappe del passaggio "dal tempo della Chiesa a quello del mercante"; un tornante fondamentale nel quale, scissa la condizione di "libertà" da quella di "necessità", si attiva una «dialettica integrale»o «superdialettica» ${ }^{8}$ che presiede, attraverso progressive sintesi di superamento tra la "scienza" e la "tecnica", ai processi di socializzazione degli individui.

\section{2. "Tempo mercificato" e implosione del socialismo}

Sciolti i nodi interpretativi di fondo e definito il quadro storico-antropologico da cui prende forma la società moderna, nei cinque successivi paragrafi (dal 5 al 9) l'autore segue il corso del "tempo del mercante" e la sua trasformazione/involuzione in "tempo mercificato" a partire dalla seconda metà del XIX secolo, allorquando la nascita dell'economia di scambio impone la creazione di una natura fittizia, il mercato, che comporta l'asservimento delle ragioni del "sapere" a quelle della "utilità". Tale mutamento, che identifica la cosiddetta "seconda rivoluzione industriale", segnerà la storia dell'umanità fino alla fine del XX secolo, grazie alla capacità della nuova organizzazione capitalistica del sistema di mantenere al proprio interno quei caratteri dialettici che consentono la nascita - e, in alcuni casi, l'affermazione - di forze di opposizione in grado di immaginare un "superamento" del capitalismo e quindi ideare un tempo storico alternativo. È dentro questo confronto che può realizzarsi l'"assalto al cielo" dell'Ottobre 1917: un «messianismo dell'utopia» ${ }^{9}$ che in breve tempo, dovendo prendere atto che, in sua assenza, sarebbe occorso costruire «il capitalismo da superare» ${ }^{10}$, opta per l'elusione del problema della "transizione al socialismo", edificando una fittizia "natura socialista" dalle caratteristiche identiche a quella contro cui aveva annunciato di ergersi.

\footnotetext{
${ }^{6}$ MARINO, Giuseppe Carlo, Un'età contro la storia, cit., p. 7.

${ }^{7}$ Cfr. LE GOFF, Jacques, Nel Medioevo: tempo della Chiesa e tempo del mercante, in ID., Tempo della Chiesa e tempo del mercante e altri saggi sul lavoro e la cultura nel Medioevo, Torino, Einaudi, 1977, pp. 3-23.

${ }^{8}$ MARINO, Giuseppe Carlo, Un'età contro la storia, cit., p. 16.

${ }^{9}$ Ibidem, p. 24.

${ }^{10}$ Ibidem, p. 25.
} 
Polo del capitale e polo del socialismo rappresentano dunque, secondo l'autore, due "false dialettiche»" che per l'intero svolgersi del "secolo breve" perseguono speculari strategie di annientamento «che ne irrigidivano e ne congelavano la contraddizione impedendone il superamento in una sintesi ${ }^{12}$ : una dialettica "inautentica" che, per ciò che riguarda l'Occidente, si invera nella soluzione del "compromesso socialdemocratico" per affermare «un'universale omologazione delle propensioni alla ricerca del benessere, con un prorompente primato assicurato al criterio dell'utilità sugli ideali e sulle stesse profferte delle ideologie» ${ }^{13}$. In un quadro siffatto si realizza, per Marino, l'implosione del cosiddetto "socialismo reale", risultato incapace di riformulare la propria ragion d'essere e di competere con gli straordinari progressi dell'Occidente nel settore elettronico-informatico, all'origine della rivoluzione in corso: una rivoluzione inedita, che recide in profondità i legami con i diversi stadi - e con i tornanti cruciali - della precedente storia dell'umanità.

Questo passaggio credo sia il più arduo e problematico dell'intera trattazione. L'apprezzabile sforzo di sintetizzare processi storici così importanti rischia qui di apparire, infatti, come una scorciatoia eccessivamente deterministica, che conduce l'autore a suggestive ma fin troppo rigide conclusioni che non tengono nel dovuto conto antefatti, decisioni e processi realizzatisi sui molteplici piani del confronto Est-Ovest. Se è vero che i progressi dell'informatica e dell'elettronica in Occidente abbiano determinato la sostanziale "chiusura" della competizione per l'incapacità del sistema sovietico di tenere il passo dell'avversario, pure non si può non evidenziare come tali sviluppi non sarebbero stati pensabili senza il sostanziale cambio di rotta nella politica economica e monetaria mondiale che diede origine alla cosiddetta "finanziarizzazione" del modo di produzione capitalistico. Mi riferisco, naturalmente, alla decisione presa nel 1971 dal presidente americano Richard Nixon di sganciare il dollaro dall'oro, provocando il crollo del sistema di cambi rigidi stabilito nel 1944 a Bretton Woods. La rivoluzione informatica di cui parla Marino è contestuale a questa scelta e si configura come uno strumento necessario alla nuova politica di finanziarizzazione dell'economia e del decentramento produttivo che funge da detonatore della crisi ormai irreversibile dell'URSS (che, è bene ricordarlo, ancora nel decennio precedente era sul punto di superare lo sviluppo nordamericano), determinando l'affermazione della dottrina neoliberale (che per lungo tempo era stata minoritaria) a scapito tanto della sinistra socialdemocratica quanto di quella terzinternazionalista.

\footnotetext{
${ }^{11}$ Ibidem, p. 27.

12 Ibidem.

${ }^{13}$ Ibidem, p. 30.
} 


\section{La libertà di consumare nella democrazia di mercato}

La seconda metà del saggio è dedicata alla contemporaneità, ovvero all'analisi delle ripercussioni del modello economico-produttivo - e del suo correlato ideologico - ormai egemone sull'umanità e sul suo sistema di relazioni e organizzazione sociale. Due fenomeni, in particolare, sono al centro della riflessione di Marino: il mutamento dell'organizzazione produttiva e i risvolti di questo sulle identità dei soggetti e, in correlazione, lo svuotamento della democrazia. Sul primo punto, l'autore sostiene che la nascita di un inedito capitalismo, il cui tratto distintivo è costituito $\mathrm{da}$ «un'alleanza organica tra la tecnica che genera altra tecnica e il denaro che, in essa e tramite essa, produce altro denaro ${ }^{14}$, ha comportato che i profitti siano conseguiti soprattutto mediante la commercializzazione di prodotti di preminente valenza tecnologica piuttosto che attraverso la produzione di beni manifatturieri industriali. Ciò, in realtà, è vero in parte, poiché i canali dell'accumulazione capitalistica, più che sostituiti, oggi sembrano essersi moltiplicati. Di fianco alla "commercializzazione" e all'investimento nei servizi, infatti, nell'epoca attuale il vettore privilegiato del salto quantico del modo di produzione capitalistico è rappresentato dall'impiego sempre più crescente di capitali nei circuiti immateriali della finanza: inedita modalità di creazione di profitto dotata di una altrettanta inedita capacità di influenzare le decisioni politiche e, di concerto con le strategie delle grandi organizzazioni sovranazionali, di determinare persino la sopravvivenza e il mantenimento di una, ancorché parziale, autonomia degli Stati. La diversificazione delle forme e degli spazi di accumulazione, però, non ha inciso - o almeno non ancora - in misura così rilevante sulla produzione di beni manifatturieri, la cui domanda, piuttosto, sembra essere aumentata in un mercato sempre più globale. A fronte di un inesorabile ampliamento delle disuguaglianze, il numero di lavoratori salariati nel mondo è, del resto, incomparabilmente più elevato rispetto alla metà del secolo scorso e svariati milioni di esseri umani - prevalentemente dei paesi asiatici, dell'America latina ma anche dell'area islamica - sono fuoriusciti dalla condizione di asservimento e povertà. Di certo, invece, è pienamente condivisibile la tesi dell'autore secondo cui i rivoluzionari mutamenti nel modo di produzione e di investimento abbiano agito sulla morfologia stessa della forza-lavoro, per un verso creando una omologazione di "valori" e necessità, per un altro favorendo processi di enorme disgregazione identitaria e di classe che hanno definito individui sostanzialmente isolati, diversi tra loro soltanto nella collocazione all'interno di un'unica gerarchia della ricchezza e liberi, in definitiva, di consumare per produrre e di produrre per consumare.

Contraltare "politico" dei processi testé descritti - e secondo elemento al centro dell'analisi di Marino - è la liquidazione della "democrazia rivoluzionaria" che l'Occidente aveva elaborato e sviluppato nel XX secolo. L'omologazione "culturale" di individui-monadi, infatti, richiede che le

\footnotetext{
${ }^{14}$ Ibidem, p. 45.
} 
formazioni politiche si conformino al verbo del "pensiero unico", annientando le loro diverse visioni del mondo e ciò che definisce il loro statuto, ovvero la valorizzazione nel "collettivo" - e per suo tramite - delle soggettività ${ }^{15}$. Naturalmente, l'annichilimento dei partiti tradizionali viene propagandato e percepito come un avanzamento in direzione di una democrazia compiuta, di cui sarebbe dimostrazione la proliferazione di movimenti-contenitore ove confluiscono prevalentemente «mutevoli stati d'animo individuali precariamente condivisi, nonché aspirazioni, slanci utopici e proteste» ${ }^{16}$. In realtà, come è possibile osservare nel caso italiano, la promessa liberazione dei soggetti individuali dalla "camicia di forza" dei soggetti collettivi, propugnata da un "pensiero unico" che si autorappresenta come "a-ideologico da risultare persino super-ideologico ${ }^{17}$, ha avuto esiti abbastanza deludenti, per quanto oltremodo devastanti. Una mole di studi pubblicati negli ultimi anni in relazione alla crisi ed alla degenerazione della democrazia ${ }^{18}$, si è incaricata di dimostrare come l'implosione delle forme organizzate della politica abbia camminato di pari passo con il tramonto della partecipazione e della passione per l'interesse generale. L'affermazione dell'«individualismo narcisista» ${ }^{19} \mathrm{si}$ configura così come l'anima di un diffuso sentimento di rabbia esasperata, spesso quasi senza oggetto, orientato a proclamare «il trionfo della Piazza sul Palazzo, il rifiuto della mediazione che è alla base della democrazia rappresentativa» e a promuovere $i$ «mass media [...] a unica voce autentica della volontà popolare, autorizzata a organizzare quotidianamente un plebiscito per giudicare l'operato di coloro ai quali la gente [...] ha provvisoriamente affidato il proprio mandato $»^{20}$. Ad indicare quanto la democrazia sia oggi quasi del tutto svuotata è la riduzione del suo esercizio alla mera consultazione elettorale, stantìa prassi ormai prevalentemente ricondotta alla logica binaria "più utile/meno utile" della mentalità tecnologica, in cui prevale la proposta che, apparendo meno ideologica, riesce ad attrarre la maggior quota possibile della "domanda". Ciò dimostra come il "trionfo della piazza" sia, in questo senso, pura finzione estetica, poiché la sterilizzazione dei corpi intermedi, lungi dall'aver allargato le maglie della democrazia, ha favorito piuttosto l'egemonia del tecnicismo. Negli ultimi trent'anni il linguaggio dell'impresa è divenuto sinonimo di modernità, estendendosi a tutta la struttura sociale, compresi i settori di studio e lavoro umanistici sempre più sottoposti al diktat delle cosiddette "competenze". Del pari, la politica si è ridotta al compito di progettare ed eseguire operazioni per gestire l'esistente, laddove il "riformismo", come giustamente afferma Marino, è venuto a significare il pallido

\footnotetext{
${ }^{15}$ Su questi aspetti è d'obbligo il rinvio a BAUMAN, Zygmunt, Modernità liquida, Roma-Bari, Laterza, 2002.

${ }^{16}$ MARINO, Giuseppe Carlo, Un'età contro la storia, cit., p. 55.

${ }^{17}$ Ibidem, p. 56.

${ }^{18}$ Per una bibliografia ragionata cfr. PONTE DI PINO, Oliviero, «La democrazia: governo della crisi o modello in crisi?», in Doppiozero, URL: < http://www.doppiozero.com/materiali/la-democrazia-governo-della-crisi-omodello-in-crisi > [consultato il 10 giugno 2018].

${ }^{19} \mathrm{Cfr}$. ORSINA, Giovanni, La democrazia del narcisismo. Breve storia dell'antipolitica, Venezia, Marsilio, 2018.

${ }^{20}$ Cfr. TARCHI, Marco, Italia populista. Dal qualunquismo a Beppe Grillo, Bologna, Il Mulino, 2015, pp. 238-239; ma cfr. anche BIANCHI, Leonardo, La gente. Viaggio nell'Italia del risentimento, Roma, Minimum Fax, 2017.
} 
tentativo di procedere ad operazioni di restyling di questo eterno e sostanzialmente immodificabile presente ${ }^{21}$.

A fronte di una crisi epocale come quella in cui siamo immersi, che è crisi economica, di relazioni e di senso, esistono certamente ancora le condizioni per una esplosione sociale che metta in discussione il paradigma di civiltà esistente, ma per individuare la miccia che faccia divampare l'incendio bisognerebbe innanzitutto sviluppare una compiuta, adeguata e credibile riflessione sui processi in corso, una elaborazione capace di svincolarsi dalle strettoie di impianti ideologici preconfezionati e al contempo in grado di proporre una nuova narrazione del nostro tempo. In tal senso, il pamphlet di Carlo Marino condensa idee importanti, stimolanti e, direi, necessarie, che possono costituire un punto di inizio. Ridefinire un nuovo "soggetto rivoluzionario" è certo impresa complessa, ma per farlo sarà irrinunciabile tornare all'origine della riflessione gramsciana sui "subalterni" e riprendere suggestioni potenti del pensiero contemporaneo, ad iniziare da quelle, attualissime, di Herbert Marcuse sulla forza «dei reietti e degli stranieri» $»^{22}$, ben sapendo, come afferma Enzo Traverso, che «le rivoluzioni non si decretano» e, soprattutto, che non tutte sono gioiose, poiché alcune volte possono configurarsi anche come una «"scommessa malinconica"»".

${ }^{21}$ MARINO, Giuseppe Carlo, Un'età contro la storia, cit., pp. 68-69.

${ }^{22}$ MARCUSE, Herbert, L'uomo a una dimensione, Torino, Einaudi, 1968 [ed. orig.: 1964], p. 165.

${ }^{23}$ TRAVERSO, Enzo, Che fine hanno fatto gli intellettuali? Conversazione con Régis Meyran, Verona, Ombre Corte, 2014, p. 103; la citazione è di BENSAÏD, Daniel, Le pari mélancolique. Métamorphoses de la politique, politique des métamorphoses, Paris, Fayard, 1997. 


\section{L'AUTORE}

Carmelo ALBANESE (1979) ha conseguito il titolo di dottore di ricerca in Storia contemporanea all'Università di Firenze con una tesi sulla lotta politica in Sicilia in età giolittiana ( $I^{\circ}$ Premio "Spadolini Nuova Antologia" - XVIII edizione). Interessato ai temi dell'antifascismo, della Resistenza e della società italiana fra le due guerre, dal 2014 collabora con l'Istituto Storico Toscano della Resistenza e dell'Età Contemporanea (ISRT). Tra le sue recenti pubblicazioni si segnalano i volumi: Leto Fratini, scultore. Percorsi esistenziali e traiettorie dell'antifascismo tra Firenze e Milano, Pisa, Pacini Editore, 2017; Storia dell'Anpi Oltrarno. Associazionismo popolare e memoria della Resistenza a Firenze, Firenze, Anpi Oltrarno, 2017.

URL: < http://www.studistorici.com/progett/autori/\#Albanese > 